\title{
anntraining
}

November 11, 2021

In this PDF file some links won't work. Find the fully featured Jupyter Notebook file on the website of Prof. Jens Flemming at Zwickau University of Applied Sciences. This work is licensed under a Creative Commons Attribution-ShareAlike 4.0 International License.

\section{Training ANNs}

We have a close look gradient descent methods and implement a complete ANN approach. Then we went on to Scikit-Learn's ANN routines.

\subsection{Gradient descent for nonlinear minimization problems}

Consider a function $h: \mathbb{R}^{p} \rightarrow \mathbb{R}$ and the corresponding minimization problem

$$
h\left(w_{1}, \ldots, w_{p}\right) \rightarrow \min _{w \in \mathbb{R}^{p}}
$$

The gradient

$$
\nabla h\left(w_{1}, \ldots, w_{p}\right)=\left[\begin{array}{c}
\frac{\partial}{\partial w_{1}} h\left(w_{1}, \ldots, w_{p}\right) \\
\vdots \\
\frac{\partial}{\partial w_{p}} h\left(w_{1}, \ldots, w_{p}\right)
\end{array}\right]
$$

is the vector of partial derivatives of $h$ with respect to all variables $w_{1}, \ldots, w_{p}$. The gradient is known to be the direction of steepest ascent. In other words, $-\nabla h(w)$ is the direction of steepest descent of $h$ at $w$.

To find a minimizer of $h$ we might start at some point $w^{(0)}$, substract the gradient $\nabla h\left(w^{(0)}\right)$ giving a new point $w^{(1)}$, substract $\nabla h\left(w^{(1)}\right)$, and so on. This way function values should become smaller step by step. The problem is that the negative gradient only provides a direction, but no information about how far we should go in this direction. Thus, we have to introduce a parameters $s_{0}, s_{1}, \ldots$ for each step controlling the step length.

For general directions we have the following algorithm: 1 . Choose a starting point $w^{(0)}$. 2. Repeat for $i=0,1, \ldots: 1$. Choose a direction $r_{i}$. 2. Choose a step length $s_{i}$. 3. Set $w^{(i+1)}=w^{(i)}+s_{i} r_{i} 4$. If $\left|w^{(i+1)}-w^{(i)}\right|$ is small enough, then stop iteration.

In the simplest case we would choose

$$
r_{i}=-\nabla h\left(w^{(i)}\right) \quad \text { and } \quad s_{i}=s
$$

with a constant step length $s>0$. With this choice we obtain the steepest descent method. It is not guaranteed to converge. Convergence means, that the stopping cirterion is satisfied after 
sufficiently many steps. Small $s$ yields higher chances for convergence, but many iterations are required. Large $s$ decreases the number of iterations, but the method may not converge.

To improve performance directions different from the negative gradient can be used. There is a long ist of sensible directions and step lengths. Details and examples will be provided next semester in the lecture series on numerical methods.

\subsection{Gradient descent for ANNs}

To apply gradient descent for training ANNs we have to compute the gradient of the objective

$$
h(w)=\frac{1}{n} \sum_{l=1}^{n}\left(f_{\text {approx }}\left(x_{l}\right)-y_{l}\right)^{2}
$$

with respect to th weight vector $w$ containing all weights of the ANN.

For each component of the gradient $\nabla_{w} h(w)$ we have

$$
\frac{\partial}{\partial w_{\kappa}} h(w)=\frac{1}{n} \frac{\partial}{\partial w_{\kappa}} \sum_{l=1}^{n}\left(f_{\text {approx }}\left(x_{l}\right)-y_{l}\right)^{2}=\frac{1}{n} \sum_{l=1}^{n} \frac{\partial}{\partial w_{\kappa}}\left(f_{\text {approx }}\left(x_{l}\right)-y_{l}\right)^{2} .
$$

Chain rule yields

$$
\frac{\partial}{\partial w_{\kappa}} h(w)=\frac{1}{n} \sum_{l=1}^{n} 2\left(f_{\text {approx }}\left(x_{l}\right)-y_{l}\right) \frac{\partial}{\partial w_{\kappa}} f_{\text {approx }}\left(x_{l}\right) .
$$

Thus,

$$
\nabla h(w)=\frac{2}{n} \sum_{l=1}^{n}\left(f_{\text {approx }}\left(x_{l}\right)-y_{l}\right) \nabla f_{\text {approx }}\left(x_{l}\right)
$$

If we take $\nabla f_{\text {approx }}\left(x_{1}\right), \ldots, \nabla f_{\text {approx }}\left(x_{n}\right)$ as columns of a matrix $G \in \mathbb{R}^{p \times n}$ with $p$ being the total number of weights and if we set

$$
y_{\text {pred }}:=\left[\begin{array}{c}
f_{\text {approx }}\left(x_{1}\right) \\
\vdots \\
f_{\text {approx }}\left(x_{n}\right)
\end{array}\right] \quad \text { and } \quad y=\left[\begin{array}{c}
y_{1} \\
\vdots \\
y_{n}
\end{array}\right]
$$

we obtain

$$
\nabla h(w)=\frac{2}{n} G\left(y_{\mathrm{pred}}-y\right)
$$

It remains to find the gradient of $f_{\text {approx }}(x)$ with respect to the weight vector $w$ for some feature vector $x$. This gradient heavily depends on the structure of the ANN.

\subsubsection{Single layer ANNs}

We compute the gradient $\nabla f_{\text {approx }}(x)$ for an ANN with only one layer. The main difficulty is to find manageable notation. We have $m$ features, $q$ regular neurons, two bias neurons and one output neuron. 


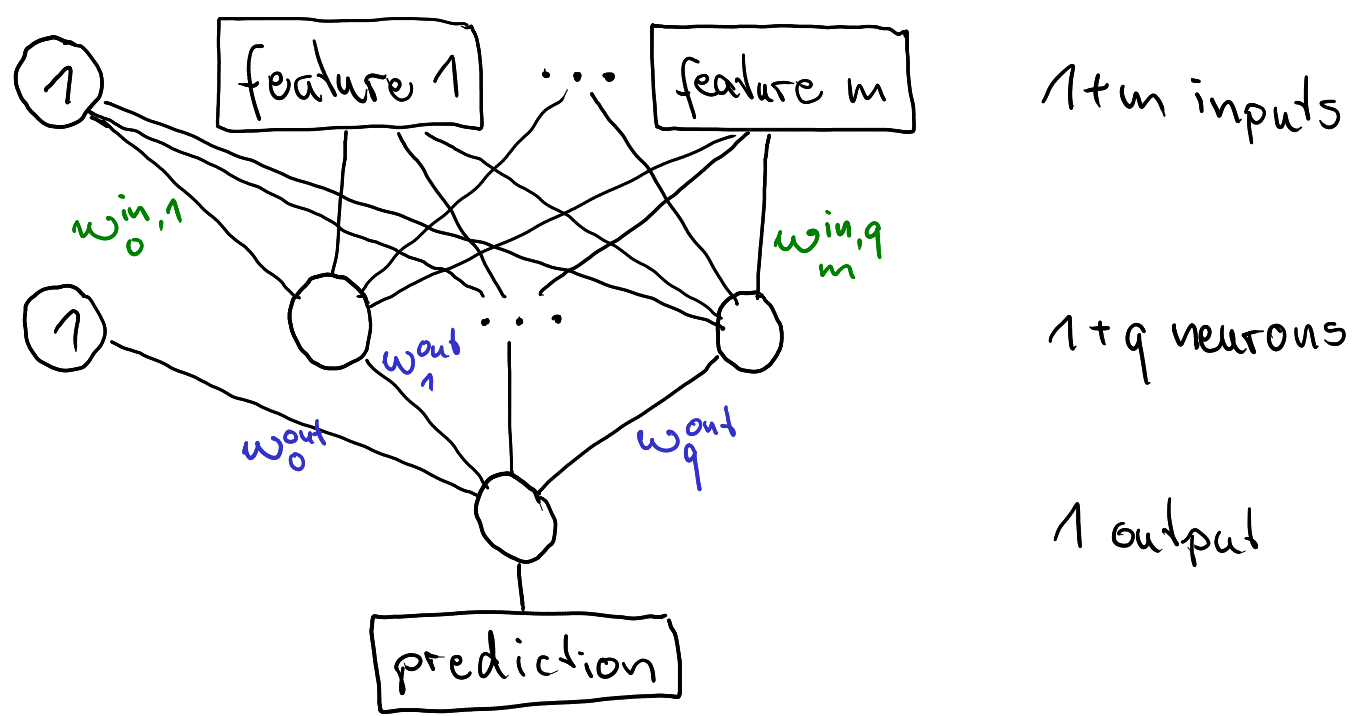

We number the regular neurons by $1, \ldots, q$. Weights of the regular neuron with number $\mu$ are denoted by $w_{0}^{\text {in }, \mu}, w_{1}^{\text {in }, \mu}, \ldots, w_{m}^{\text {in }, \mu}$, where $w_{0}^{\text {in, }, \mu}$ is the weight of the bias input and the others are for the $m$ feature inputes. Weights of the output neuron are denoted by $w_{0}^{\text {out }}, w_{1}^{\text {out }}, \ldots, w_{q}^{\text {out }}$. Again, $w_{0}^{\text {out }}$ is for the bias input and the others are for the inputs from the $q$ regular neurons. Activation functions are $g_{\text {in }}$ for all regular neurons and $g_{\text {out }}$ for the output neuron.

With this notation we have

$$
w=\left[\begin{array}{llllllllll}
w_{0}^{\text {in }, 1} & \cdots & w_{m}^{\text {in }, 1} & \cdots & w_{0}^{\text {in }, q} & \cdots & w_{m}^{\text {in }, q} & w_{0}^{\text {out }} & \cdots & w_{q}^{\text {out }}
\end{array}\right]^{\mathrm{T}} \in \mathbb{R}^{(m+1) q+q+1}
$$

for the weight vector and

$$
f_{\text {approx }}(x)=g_{\text {out }}\left(w_{0}^{\text {out }}+\sum_{\mu=1}^{q} w_{\mu}^{\text {out }} g_{\text {in }}\left(w_{0}^{\text {in }, \mu}+\sum_{k=1}^{m} w_{k}^{\text {in }, \mu} x^{(k)}\right)\right)
$$

for the hypothesis. Chain rule yields

$$
\begin{aligned}
& \frac{\partial}{\partial w_{0}^{\text {in }, \mu}} f_{\text {approx }}(x)=w_{\mu}^{\text {out }} g_{\text {in }}^{\prime}\left(w_{0}^{\text {in }, \mu}+\sum_{k=1}^{m} w_{k}^{\text {in }, \mu} x^{(k)}\right) g_{\text {out }}^{\prime}\left(w_{0}^{\text {out }}+\sum_{\tilde{\mu}=1}^{q} w_{\tilde{\mu}}^{\text {out }} g_{\text {in }}\left(w_{0}^{\text {in }, \tilde{\mu}}+\sum_{k=1}^{m} w_{k}^{\text {in }, \tilde{\mu}} x^{(k)}\right)\right), \\
& \frac{\partial}{\partial w_{k}^{\text {in }, \mu}} f_{\text {approx }}(x)=x^{(k)} w_{\mu}^{\text {out }} g_{\text {in }}^{\prime}\left(w_{0}^{\text {in }, \mu}+\sum_{\tilde{k}=1}^{m} w_{\tilde{k}}^{\text {in }, \mu} x^{(\tilde{k})}\right) g_{\text {out }}^{\prime}\left(w_{0}^{\text {out }}+\sum_{\tilde{\mu}=1}^{q} w_{\tilde{\mu}}^{\text {out }} g_{\text {in }}\left(w_{0}^{\text {in }, \tilde{\mu}}+\sum_{\tilde{k}=1}^{m} w_{\tilde{k}}^{\text {in }, \tilde{\mu}} x^{(\tilde{k})}\right)\right)
\end{aligned}
$$

and

$$
\begin{gathered}
\frac{\partial}{\partial w_{0}^{\text {out }}} f_{\text {approx }}(x)=g_{\text {out }}^{\prime}\left(w_{0}^{\text {out }}+\sum_{\mu=1}^{q} w_{\mu}^{\text {out }} g_{\text {in }}\left(w_{0}^{\text {in }, \mu}+\sum_{k=1}^{m} w_{k}^{\text {in }, \mu} x^{(k)}\right)\right), \\
\frac{\partial}{\partial w_{\mu}^{\text {out }}} f_{\text {approx }}(x)=g_{\text {in }}\left(w_{0}^{\text {in }, \mu}+\sum_{k=1}^{m} w_{k}^{\text {in }, \mu} x^{(k)}\right) g_{\text {out }}^{\prime}\left(w_{0}^{\text {out }}+\sum_{\tilde{\mu}=1}^{q} w_{\tilde{\mu}}^{\text {out }} g_{\text {in }}\left(w_{0}^{\text {in }, \tilde{\mu}}+\sum_{k=1}^{m} w_{k}^{\text {in }, \tilde{\mu}} x^{(k)}\right)\right) .
\end{gathered}
$$


Introducing activations

$$
a^{\text {in }, \mu}:=w_{0}^{\text {in }, \mu}+\sum_{k=1}^{m} w_{k}^{\text {in }, \mu} x^{(k)}
$$

for the regular neurons and

$$
a^{\text {out }}:=w_{0}^{\text {out }}+\sum_{\mu=1}^{q} w_{\mu}^{\text {out }} g_{\text {in }}\left(a^{\text {in }, \mu}\right)
$$

for the output neuron, we may rewrite those formulas as

$$
\begin{gathered}
\frac{\partial}{\partial w_{0}^{\text {in }, \mu}} f_{\text {approx }}(x)=w_{\mu}^{\text {out }} g_{\text {in }}^{\prime}\left(a^{\text {in }, \mu}\right) g_{\text {out }}^{\prime}\left(a^{\text {out }}\right), \\
\frac{\partial}{\partial w_{k}^{\text {in }, \mu}} f_{\text {approx }}(x)=x^{(k)} w_{\mu}^{\text {out }} g_{\text {in }}^{\prime}\left(a^{\text {in }, \mu}\right) g_{\text {out }}^{\prime}\left(a^{\text {out }}\right)
\end{gathered}
$$

and

$$
\begin{gathered}
\frac{\partial}{\partial w_{0}^{\text {out }}} f_{\text {approx }}(x)=g_{\text {out }}^{\prime}\left(a^{\text {out }}\right), \\
\frac{\partial}{\partial w_{\mu}^{\text {out }}} f_{\text {approx }}(x)=g_{\text {in }}\left(a^{\text {in }, \mu}\right) g_{\text {out }}^{\prime}\left(a^{\text {out }}\right) .
\end{gathered}
$$

To get the gradient $\nabla h(w)$ we have to do the following: 1 . Calculate all activations for all feature vectors $x_{1}, \ldots, x_{n}$. 2. Calculate predictions for all feature vectors $x_{1}, \ldots, x_{n}$ (based on activations from step 1). 3. Built the gradient matrix $G$ (based on activations from step 1). 4. Calculate $\nabla h(w)$ from predictions and gradient matrix (see above).

\subsubsection{Multilayer ANNs}

We compute the gradient $\nabla f_{\text {approx }}(x)$ for an ANN with $L \geq 2$ layers (excluding the output layer). Above we considered the special case $L=1$. For general $L \in \mathbb{N}$ notation is slightly more difficult.

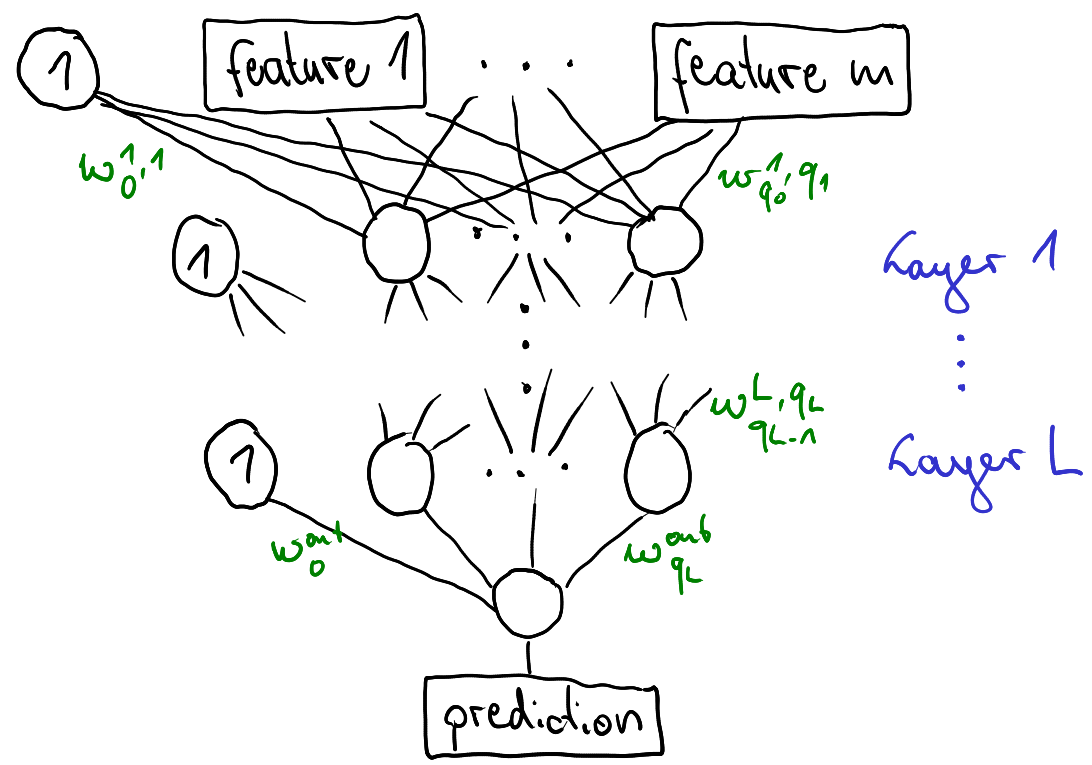


We number the layers from 1 (connected to ANN inputs) to $L$ (connected to output neuron). Layer 1 has $q_{1}$ neurons, layer 2 has $q_{2}$ neurons, and so on. We set $q_{0}:=m$ to be the number of features (inputs to layer 1). Weights are denoted by $w_{\nu}^{\lambda, \mu}$ with $\lambda=1, \ldots, L$ for the layer, $\mu=1, \ldots, q_{\lambda}$ for the neuron in the layer, $\nu=0,1, \ldots, q_{\lambda-1}$ for the inputs of the neuron ( 0 for bias neuron plus $q_{\lambda-1}$ regular neurons in previous layer). Weights of the output neuron are $w_{0}^{\text {out }}, \ldots, w_{q_{L}}^{\text {out }}$. Activation functions are denoted layerwise by $g_{1}, \ldots, g_{L}$ and $g_{\text {out }}$.

We have

$$
w=\left[\begin{array}{llllllllll}
w_{0}^{1,1} & \cdots & w_{q_{0}}^{1, q_{1}} & \cdots & w_{0}^{L, 1} & \cdots & w_{q_{L-1}}^{L, q_{L}} & w_{0}^{\text {out }} & \cdots & w_{q_{L}}^{\text {out }}
\end{array}\right]^{\mathrm{T}} \in \mathbb{R}^{p}
$$

with

$$
p=1+q_{L}+\sum_{\lambda=1}^{L} q_{\lambda}\left(1+q_{\lambda-1}\right)
$$

for the weight vector.

For each neuron corresponding activations are defined as follows:

$$
\begin{gathered}
a^{1, \mu}:=w_{0}^{1, \mu}+\sum_{\nu=1}^{q_{0}} w_{\nu}^{1, \mu} x^{(\nu)} \quad \text { for } \quad \mu=1, \ldots, q_{1}, \\
a^{\lambda, \mu}:=w_{0}^{\lambda, \mu}+\sum_{\nu=1}^{q_{\lambda-1}} w_{\nu}^{\lambda, \mu} g_{\lambda-1}\left(a^{\lambda-1, \nu}\right) \quad \text { for } \quad \mu=1, \ldots, q_{\lambda} \quad \text { and } \quad \lambda=2, \ldots, L, \\
a^{\text {out }}:=w_{0}^{\text {out }}+\sum_{\nu=1}^{q_{L}} w_{\nu}^{\text {out }} g_{L}\left(a^{L, \nu}\right) .
\end{gathered}
$$

With this notation the hypothesis is

$$
f_{\text {approx }}(x)=g_{\text {out }}\left(a^{\text {out }}\right) .
$$

Partial derivatives with respect to the weights of the output neuron are

$$
\begin{gathered}
\frac{\partial}{\partial w_{0}^{\text {out }}} f_{\text {approx }}(x)=g_{\text {out }}^{\prime}\left(a^{\text {out }}\right) \frac{\partial}{\partial w_{0}^{\text {out }}} a^{\text {out }}=g_{\text {out }}^{\prime}\left(a^{\text {out }}\right), \\
\frac{\partial}{\partial w_{\nu}^{\text {out }}} f_{\text {approx }}(x)=g_{\text {out }}^{\prime}\left(a^{\text {out }}\right) \frac{\partial}{\partial w_{\nu}^{\text {out }}} a^{\text {out }}=g_{\text {out }}^{\prime}\left(a^{\text {out }}\right) g_{L}\left(a^{L, \nu}\right) \quad \text { for } \quad \nu=1, \ldots, q_{L} .
\end{gathered}
$$

For the other neurons we have

$$
\frac{\partial}{\partial w_{\nu}^{\lambda, \mu}} f_{\text {approx }}(x)=g_{\text {out }}^{\prime}\left(a^{\text {out }}\right) \frac{\partial}{\partial w_{\nu}^{\lambda, \mu}} a^{\text {out }}=g_{\text {out }}^{\prime}\left(a^{\text {out }}\right)\left(\sum_{\tilde{\nu}=1}^{q_{L}} w_{\tilde{\nu}}^{\text {out }} g_{L}^{\prime}\left(a^{L, \tilde{\nu}}\right) \frac{\partial}{\partial w_{\nu}^{\lambda, \mu}} a^{L, \tilde{\nu}}\right) \quad \text { for } \quad \nu=0,1, \ldots, q_{\lambda-1}
$$

We see that we need the partial derivatives of the activations at layer $L$. These will depend on the derivatives of the activations at previous layers. Thus, we calculate all partial derivatives of all activations. Consider a weight $w_{\nu}^{\lambda, \mu}$. This weight has now influence on activations at layers above layer $\lambda$ and it also has no influence on activations of neurons at layer $\lambda$ other than neuron $\mu$. So 
corresponding derivatives are zero. For activations $a^{\lambda, \mu}$ (neuron the weight belongs to) we get an explicit formula. For layers below layer $\lambda$ we obtain recursive formulas:

$$
\begin{aligned}
\frac{\partial}{\partial w_{\nu}^{\lambda, \mu}} a^{\tilde{\lambda}, \tilde{\mu}}= & \frac{\partial}{\partial w_{\nu}^{\lambda, \mu}}\left(w_{0}^{\tilde{\lambda}, \tilde{\mu}}+\sum_{\tilde{\nu}=1}^{q_{\tilde{\lambda}-1}} w_{\tilde{\nu}}^{\tilde{\lambda}, \tilde{\mu}} g_{\tilde{\lambda}-1}\left(a^{\tilde{\lambda}-1, \tilde{\nu}}\right)\right) \\
= & \begin{array}{ll}
0, & \text { if } \tilde{\lambda}<\lambda, \\
0, & \text { if } \tilde{\lambda}=\lambda, \tilde{\mu} \neq \mu, \\
1, & \text { if } \tilde{\lambda}=\lambda, \tilde{\mu}=\mu, \nu=0, \\
g_{\lambda-1}\left(a^{\lambda-1, \nu}\right), & \text { if } \tilde{\lambda}=\lambda, \tilde{\mu}=\mu, \nu>0, \\
\sum_{\tilde{\lambda}-1} w_{\tilde{\lambda}}^{\tilde{\lambda}, \tilde{\mu}} g_{\tilde{\lambda}-1}^{\prime}\left(a^{\tilde{\lambda}-1, \tilde{\nu}}\right) \frac{\partial}{\partial w_{\nu}^{\lambda, \mu}} a^{\tilde{\lambda}-1, \tilde{\nu}}, & \text { if } \tilde{\lambda}>\lambda .
\end{array}
\end{aligned}
$$

To get partial derivatives for activations on layer $L$ with respect to a weight at layer $\lambda$ we first have to calculate all derivatives for activations at layer $\lambda$, then at layer $\lambda+1$ and so on until we reach layer $L$. Partial derivatives at layer $L$ then yield the desired partial derivative of the ANN's output.

\subsubsection{Weight initialization}

For the gradient descent algorithm we need to choose a starting guess. That is we have to choose initial values for all weights. We could set all weights to zero, but then all neurons in a layer would get identical input, leading to identical partial derivatives in the gradient. Thus, each layer would behave like only one single neuron. To brake this symmetry one chooses small random numbers as inital weights. All neurons will have different contributions to the ANN's output and gradient descent will favor some neurons and some neurons will become less influencial.

We have to keep in mind that gradient descent is likely to converge to a local minimum or, even worse, a stationary point close to the starting point. Thus, different sets of initial weights may yield different training results.

\subsubsection{Input standardization}

Training data should be standardized to equalize numeric ranges of different features. If there would be a feature with much higher values than the others, then this feature would have much more influence on the initial activations of the neurons. Thus, corresponding weights will have large components in the gradient and will undergo heavy manimulation whereas the other weights change only slightly in each gradient descent step.

\subsubsection{Stochastic and mini-batch training}

In each step of the gradient descent method we need to access the whole data set. Each column of the gradient matrix $G$ corresponds to one sample. For large data sets this approach consumes to many resources. If the data set does not fit into memory, then we cannot use the algorithm.

A much more efficient approach is to use only one sample per step. Then the gradient matrix $G$ has only one column and we save lots of resources. For each gradient descent step we randomly choose a different sample. Thus, all samples will have influence on the training result as before. This approach is known as stochastic gradient descent. Another advantage is that if the training 
data set grows during training, then we can integrate newly arrived data directly into the training process. This is known as online learning.

Mini-batch gradient descent is a mixture of both approaches. We split the training data set into a number of disjpint subsets and use a different subset in each gradient descent step. Compared to the full data approach we save resources, but each sample has more influence on the result. If the mini-batches are chosen randomly, then this method sometimes is refered to as stochastic gradient descent, too.

\subsubsection{How to choose step length?}

If the step length is small, then there is a good chance to find a minimizer of the objective function, but convergence will be slow. If the step length is large, then we will be relatively close to a minimizer after few descent steps, but the iterates will overshoot the minimizer. Thus, there will be no convergence.

There exist several strategies to choose the step length. In principle, step length for training is a hyperparameter of an ANN. We could try different step lengths and look at the prediction quality of the resulting ANN. Another strategy is to start with large step length and to decrease it during iteration. The idea is to get close to a minimizer within few iterations. Then the minimizer is approached slowly to avoid overshooting. There exist several other proposals for step length schedules and there are mathematically justified step length selection rules, too.

\subsubsection{Momentum methods}

Steepest descent is only one variant of gradient descent methods. Steepest descent is the simplest and straight forward, but suffers from slow convergence and from getting trapped at saddle points. An imporvement is to add momentum. Here, momentum is to be understood in the sense of physics: a ball rolling down a curvy hill does not follow the direction of steepest descent, but, due to its momentum, overshoots curves slightly and will cross small dents without problems. Thus, it will not get trapped by flat local minima or saddle points.

From the mathematical point of view adding momentum means that the step direction not only depends on the current gradient, but also on previous gradients. Usually only the gradient at the previous iterate is used in addition. There are different concrete realizations of the momentum idea out there.

\subsection{Implementation from scratch}

Later on we will use specialized Python modules for defining and training ANNs. But to gain greater insight into ANNs we implement the code for a multi-layered feedforward net from scratch.

We first define a class for representing ANNs and then we write a script implementing the steepest descent method.

[2]: import numpy as $\mathrm{np}$

import matplotlib.pyplot as plt

import mpl_toolkits.mplot3d as plt3d

$r n g=n p \cdot r a n d o m \cdot d e f a u l t \_r n g(0)$ 
[3] :

class LayeredFeedforwardANN:

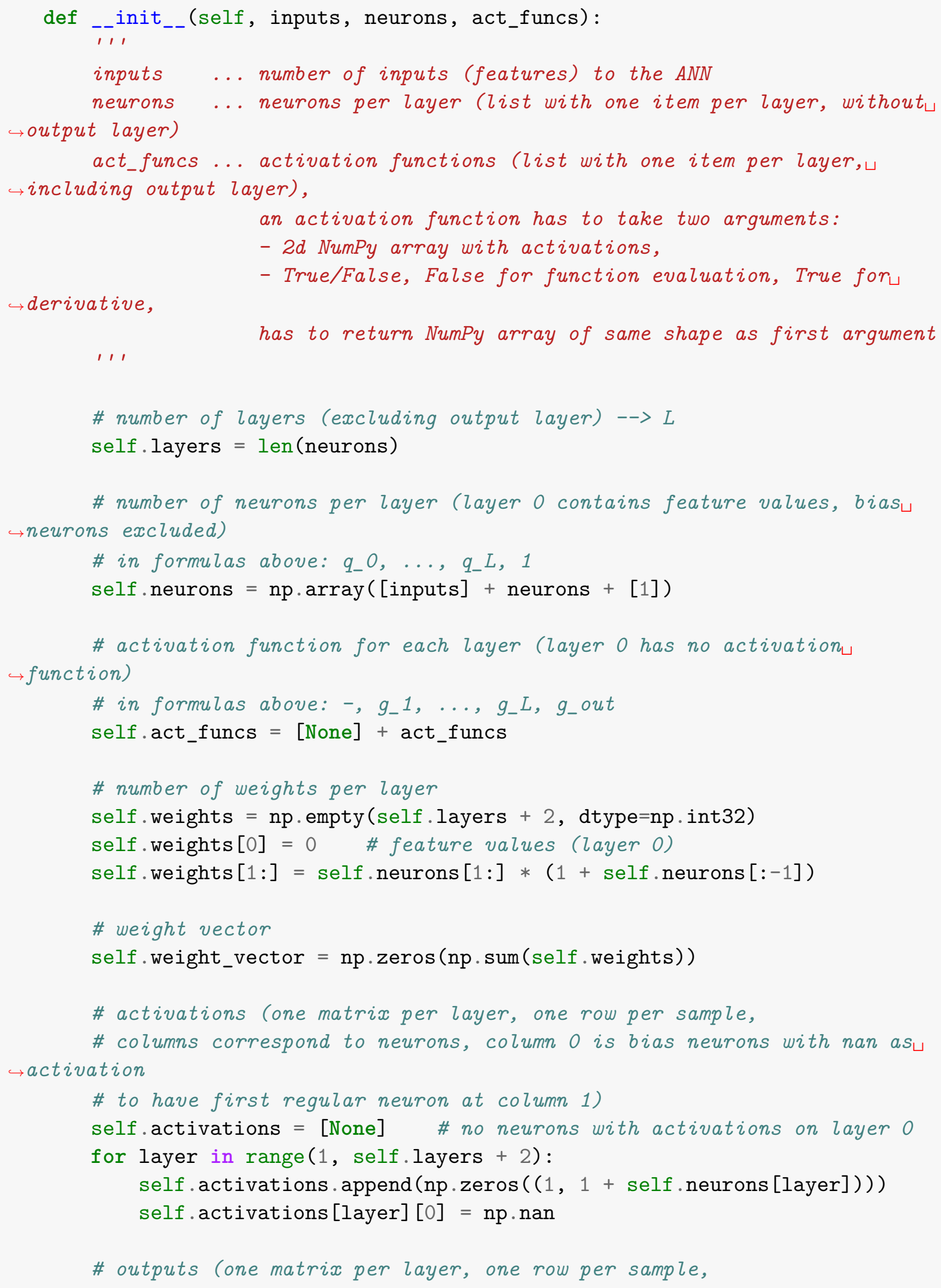




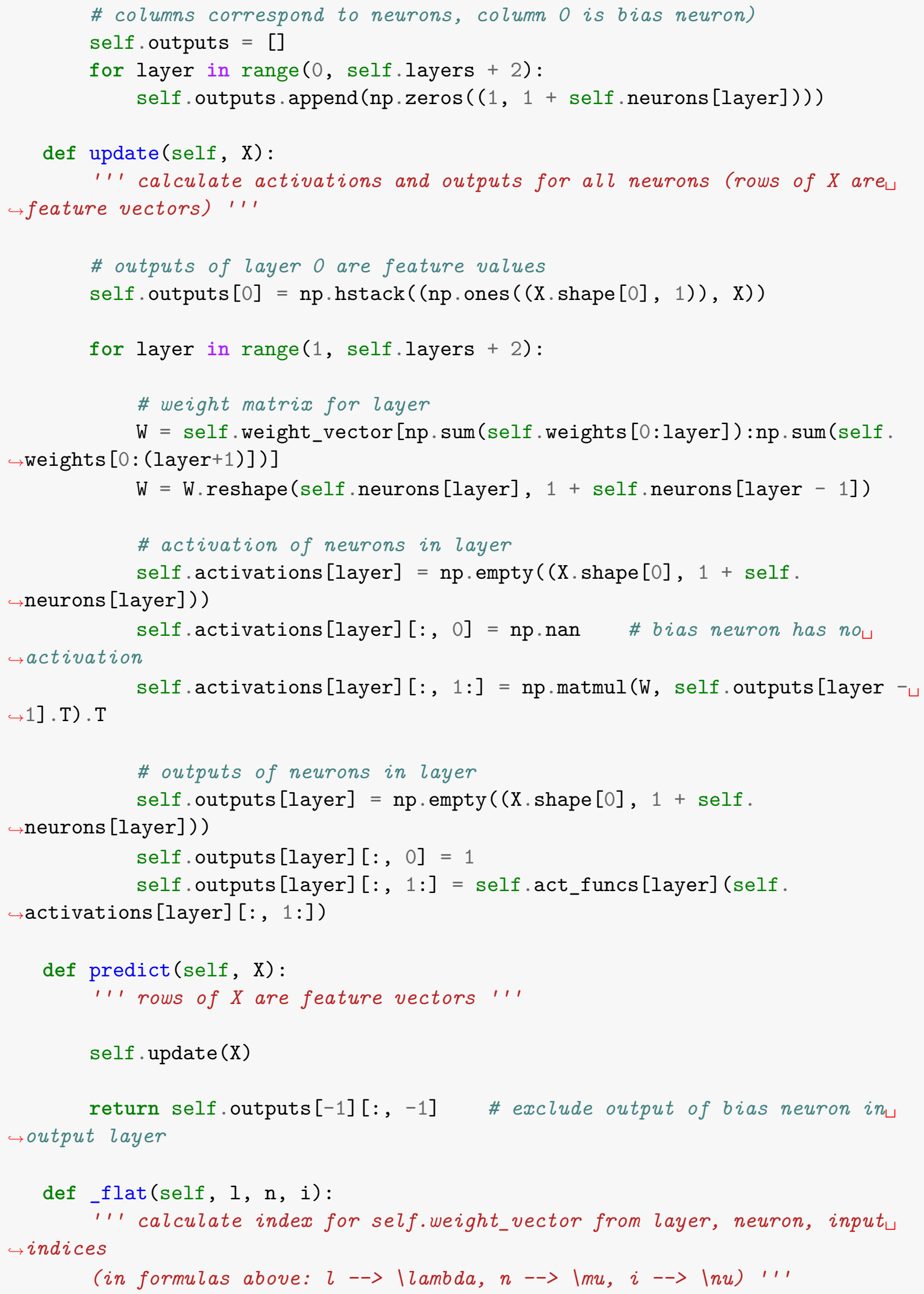




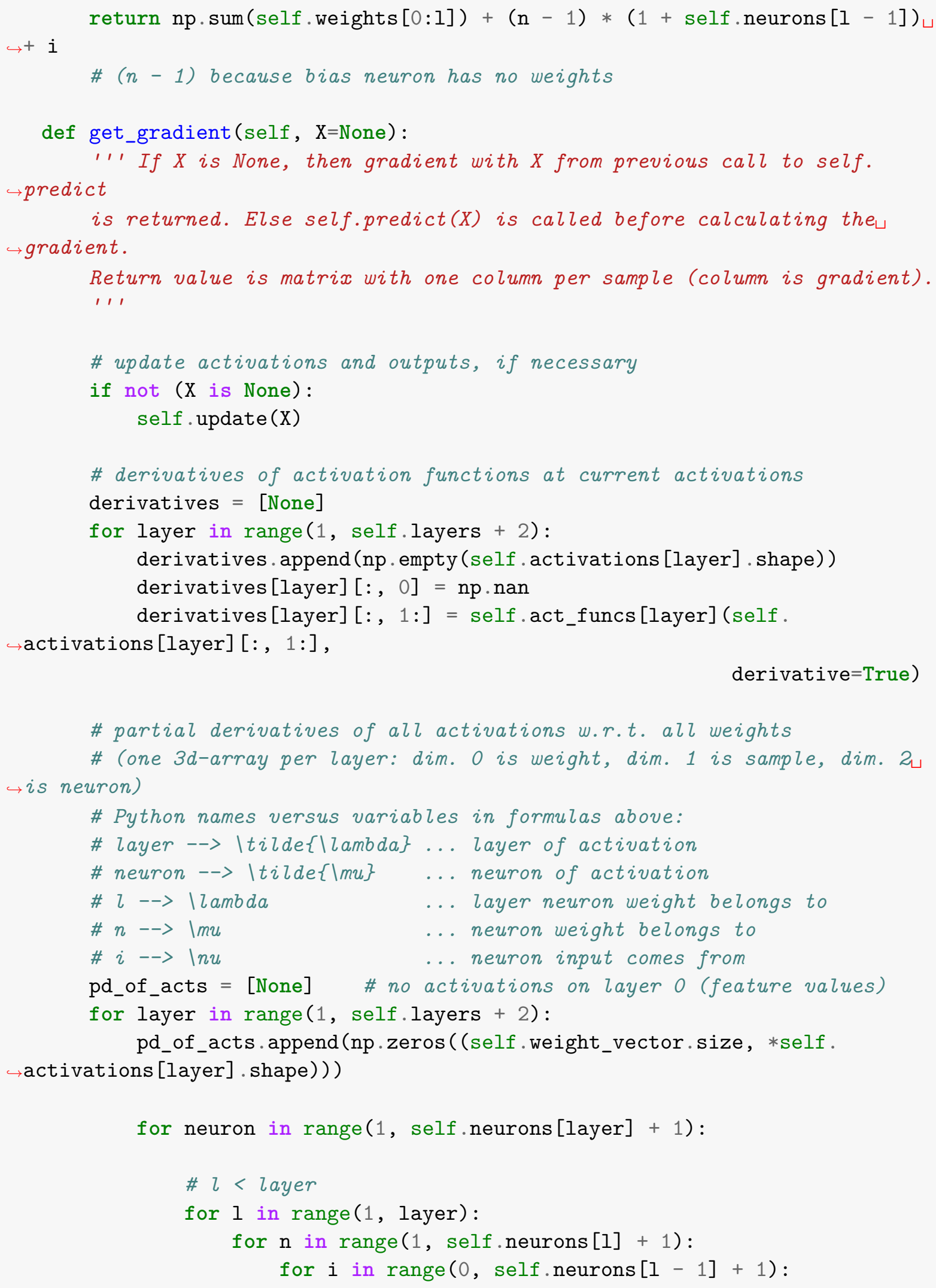




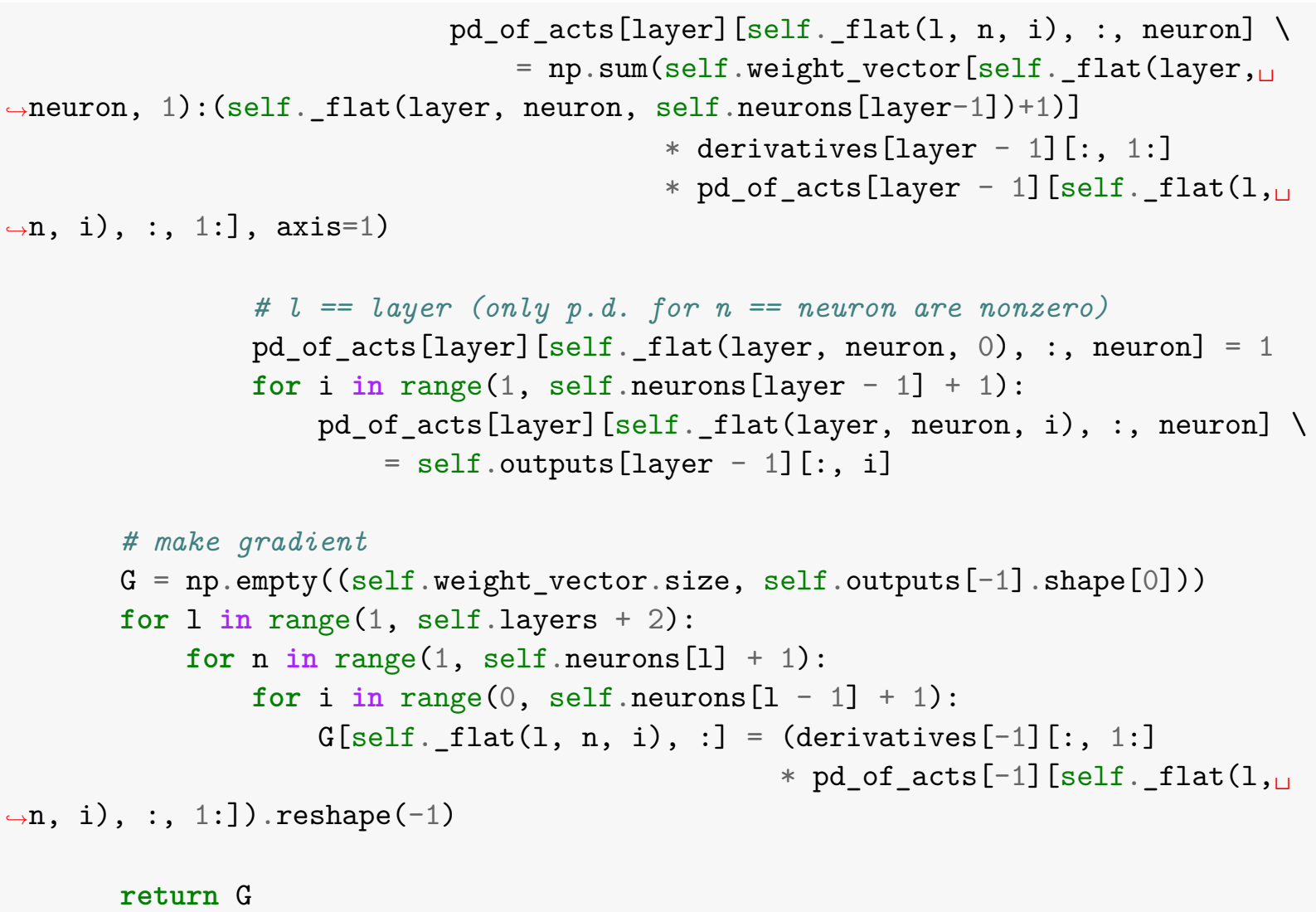

We need some activation functions. Linear activation is used for the output neuron. The others are for the hidden neurons.

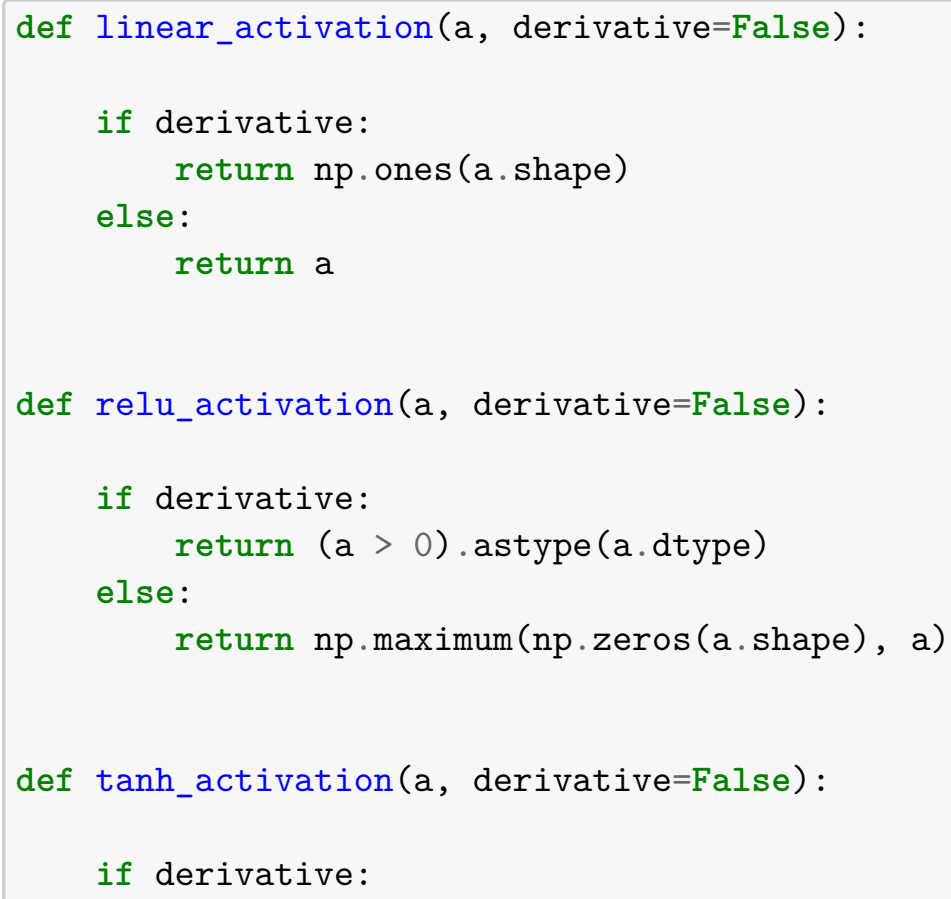


else:

return $1-\mathrm{np} \cdot \tanh (\mathrm{a}) * * 2$

return np.tanh(a)

To test our ANN code we simulate some data with two features. We only use two features for testing, because a function on $\mathbb{R}^{2}$ can be visualized as $3 \mathrm{~d}$ plot.

[5]: \%matplotlib notebook

def $\operatorname{truth}(\mathrm{x} 1, \mathrm{x} 2)$ :

return $\mathrm{np} \cdot \sin (\mathrm{np} \cdot \mathrm{pi} * \mathrm{x} 1)+\mathrm{x} 2 * * 2$

n_grid $=50 \quad$ \# grid point per axis for plotting

$\mathrm{x} 1=\mathrm{np}$.linspace $\left(-1,1, \mathrm{n}_{-}\right.$grid $)$

$\mathrm{x} 2$ = np.linspace $\left(-1,1, \mathrm{n}_{\text {_grid }}\right)$

[grid_x1, grid_x2] = np.meshgrid(x1, x2)

grid_truth $=$ truth(grid_x1, grid_x2)

fig = plt.figure ( )

ax = fig.add_subplot (111, projection='3d')

ax.plot_surface(grid_x1, grid_x2, grid_truth, color='b')

plt.show()

<IPython.core.display. Javascript object>

<IPython.core.display.HTML object>

From the true function (which is unknown in practise) we draw samples for training the ANN. To come closer to real data we add some random noise.

[6] : \%matplotlib notebook

n_samples $=100$

noise_level $=0.01$

$X=$ rng.uniform $(-1,1$, (n_samples, 2))

$\mathrm{y}=\operatorname{truth}(\mathrm{X}[:, 0], \mathrm{X}[:, 1])+\operatorname{rng} \cdot \operatorname{normal}\left(0\right.$, noise_level, $\mathrm{n}_{-}$samples $)$

fig = plt.figure ()

ax = fig.add_subplot (111, projection='3d')

ax.scatter(grid_x1, grid_x2, grid_truth, $c=' b ', s=1$ )

ax.scatter $\left(X[:, 0], X[:, 1], y, c=r^{\prime}, s=7\right)$

plt.show()

<IPython.core.display. Javascript object>

<IPython.core.display.HTML object>

We implement gradient descent using all training samples in each gradient step. 


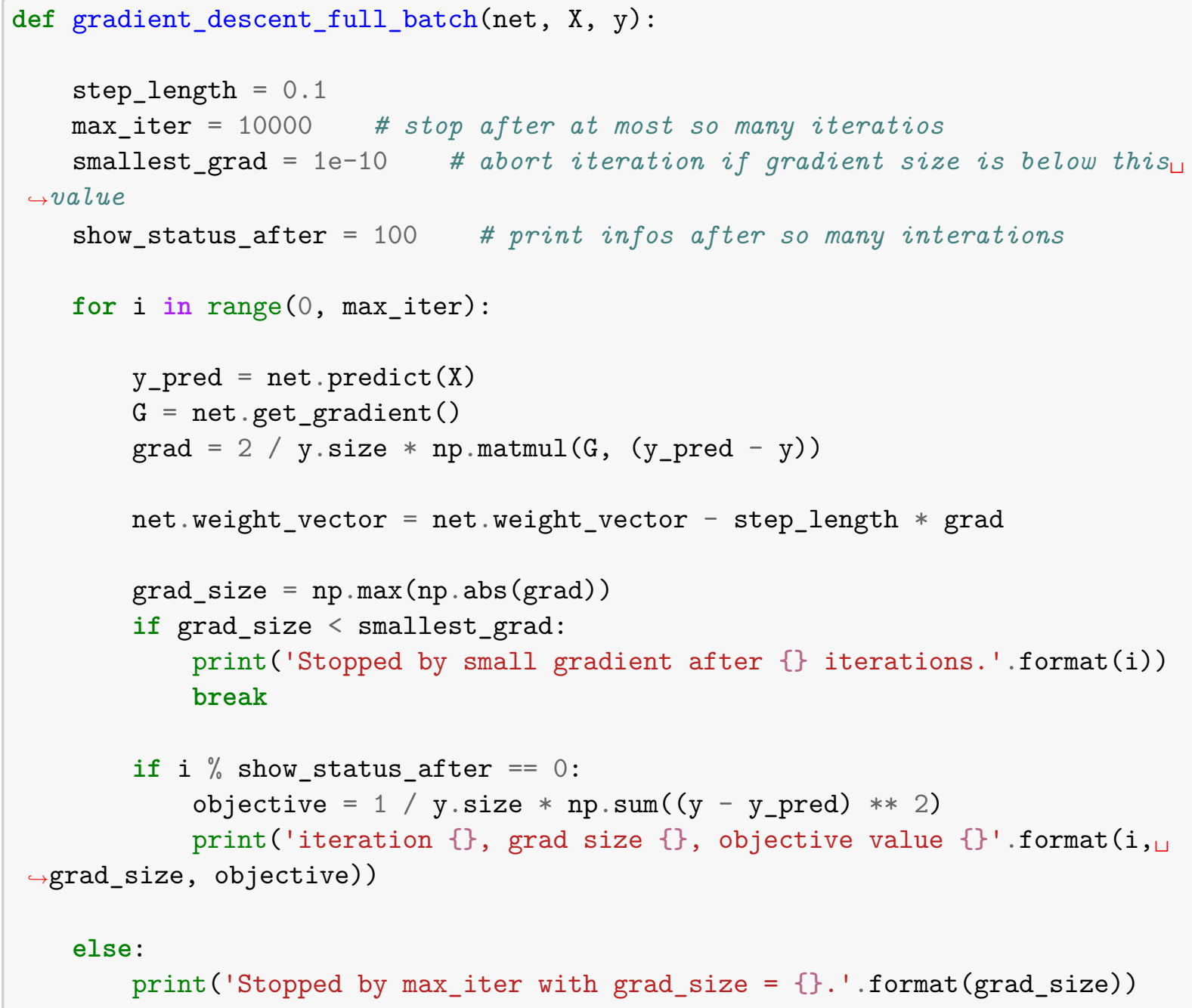

Now we define an ANN and start training.

\section{[ ]: \%matplotlib notebook}

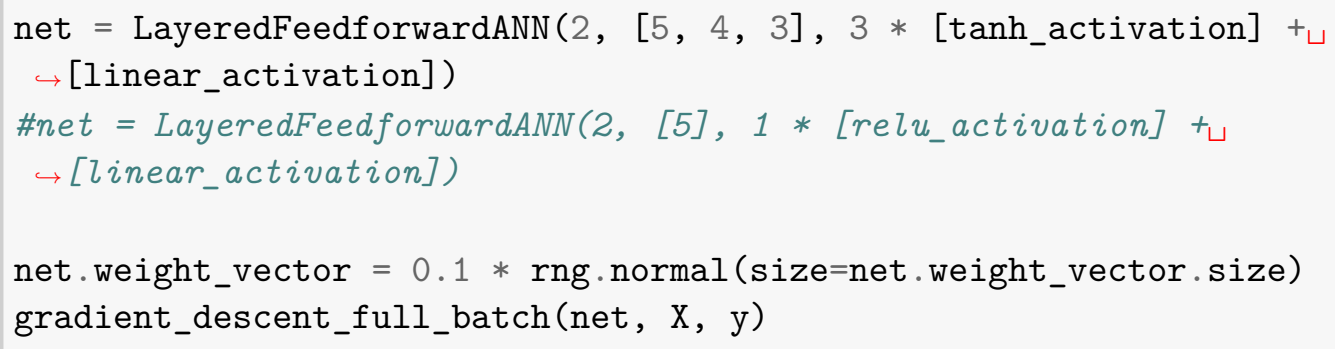

Now the net is trained and we may use it for predicting the target variable for arbitrary data.

[11]: grid_pred $=$ net.predict (np. concatenate ( $\operatorname{grid} x 1 \cdot \operatorname{reshape}(-1,1), \operatorname{grid} \mathrm{x} 2$.

$\hookrightarrow$ reshape $(-1,1))$, axis $=1)$ )

fig = plt.figure()

$\mathrm{ax}=$ fig.add_subplot(111, projection='3d') 
ax.scatter (grid_x1, grid_x2, grid_truth, $c=1 b$ ', $s=1$, label='truth')

ax.scatter (grid_x1, grid_x2, grid_pred, $c=' g^{\prime}, \mathbf{s}=7$, label='hypothesis')

ax.scatter $\left(X[:, 0], X[:, 1], y, c={ }^{\prime}{ }^{\prime}, s=20\right.$, label='training data')

ax.legend ()

plt.show()

<IPython.core.display.Javascript object>

<IPython.core.display.HTML object>

\subsection{ANNs with Scikit-Learn}

Scikit-Learn supports layered feedworward ANNs, too. Sometimes, for instance in Scikit-Learn, thay are called multi-layer perceptrons or MLPs for short. Corresponding class is MLPRegressor in sklearn.neural_network. Scikit-Learn's implementation is more efficient than ours above, but not intended for training large scale ANNs. For small and medium sized ANNs it's okay.

Usage is identical to other Scikit-Learn regressors: create an object of type MLPRegressor, call fit, and then predict. Different training algorithms are offered including gradient descent with full batch and mini-batches as well as online learning. For other algorithms have a look at the documentation. Regularization is included, too.

[]$: \# X, y$ from above

import sklearn.neural_network as neural_network

reg $=$ neural_network.MLPRegressor (hidden_layer_sizes=(5, 4, 3), activation='relu',

verbose=True) \#print status information

$\operatorname{reg} \cdot f i t(X, y)$

Now the net is trained and we may use it for prediction.

[13]: \#grid_x1, grid_x2, grid_truth from above

grid_pred $=$ reg.predict $\left(n p \cdot \operatorname{concatenate}\left(\left(\operatorname{grid} \_x 1 \cdot \operatorname{reshape}(-1,1), \operatorname{grid} x 2\right.\right.\right.$.

$\hookrightarrow$ reshape $(-1,1))$, axis $=1)$ )

fig = plt.figure ()

$a x=f i g \cdot a d d \_s u b p l o t(111$, projection='3d') 


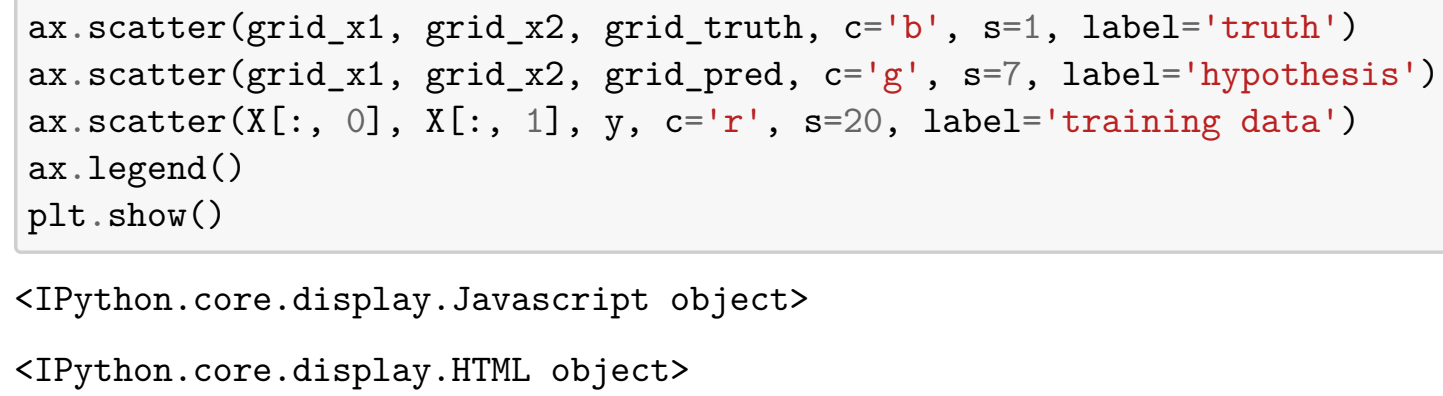

Scikit-Learn provides access to several parameters of the ANN and of the training phase. For example, we may inspect the loss curve, that is, the loss (value of objective function) for each iteration. See documentation for more.

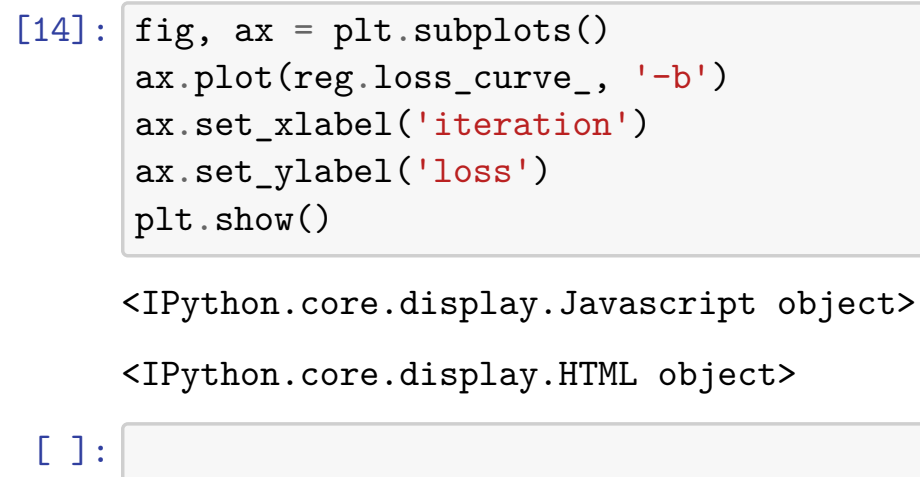

